\title{
Laparoscopic Roux-en-Y gastric bypass
}

\author{
Cüneyt Kayaalp, ${ }^{1}$ Fatih Sümer, ${ }^{1}$ Aslan Abdullayev² \\ 'Department of General Surgery, Inonu University Faculty of Medicine, Malatya, Turkey \\ ${ }^{2}$ Department of Surgery, Azerbaijan University Faculty of Medicine, Baku, Azerbaijan
}

\begin{abstract}
Total of 484 bariatric procedures, predominantly $(97.3 \%, 471$ cases) Roux-en-Y gastric bypass, were performed between March 2006 and December 2014. Bariatric program at this facility began with open Rouxen-Y gastric bypass and, in time, progressed to routine laparoscopy. After several modifications, technique was mainly standardized, and education program for laparoscopic Roux-en-Y gastric bypass was launched in June 2013. Five surgeons were educated with full, hands-on experience during the last 18 months of the program. Aim of this report was to summarize daily practice in bariatric surgery, primarily focusing on this program. Preoperative/postoperative care, and some tips and tricks related to technique of laparoscopic Roux-en-Y gastric bypass were included.

Keywords: Obesity; Roux-en-Y gastric bypass; surgical technique; preoperative care; postoperative care.
\end{abstract}

\section{Introduction}

It is difficult to say which bariatric procedure is now the most common in the world, but we can clearly say that Roux-en-Y gastric bypass is still one of the most preferred procedures. ${ }^{[1]}$ In general, published follow-up times of Roux-en-Y gastric bypass series are longer than the comparatively new developed techniques such as sleeve gastrectomy, mini-gastric bypass and gastric plication, and the cumulative data about Roux-en-Y gastric bypass is greater. While these newly developed techniques are becoming more popular among bariatric procedures, the old peers of Roux-en-Y gastric bypass, banding, and biliopancreatic diversion are losing their supporters in time with their less effectiveness or high morbidity. Some less extensive procedures have the increased risk of ineffective weight loss and/or not improving obesity related co-morbidities. On the other hand, some more complicated but more effective procedures result in severe postoperative morbidities that can be managed with difficulty. Rouxen-Y gastric bypass still maintains its popularity in most bariatric centers and it is our belief that its popularity is derived from the good balance between its effectiveness and morbidities.

\section{Preoperative Work Up}

Indications of Roux-en-Y gastric bypass for obesity do not differ from other bariatric procedures. Body mass index of at least $40 \mathrm{~kg} / \mathrm{m}^{2}$ or a body mass index of at least $35 \mathrm{~kg} /$ $\mathrm{m}^{2}$ with significant associated medical illness is the first requirement for patient selection. Secondly, potential patients must have tried other weight loss therapies such as dietary and/or regular activity, and all those efforts should 
have failed. Patients should be psychologically stable and free of substance abuse. Generally to say, patients aged between 18 and 60 are accepted, but we have some exceptions in selected cases. A complete medical evaluation is inevitable before surgery. Complete blood count, biochemical analysis, chest X-ray, electrocardiography, and abdominal ultrasound are our routine preoperative tests. Upper gastrointestinal endoscopy is performed selectively for symptomatic or age $50<$ patients..$^{[2]}$ Our obesity group, consisting the endocrinology and metabolism department, also evaluates the patients preoperatively, and they ask for some additional tests when necessary. Preoperative anesthesia and cardiology consultations are performed routinely, but other consultations and advanced test are prompted when necessary. All patients and their test results are again checked by the obesity team during obesity council on every Tuesday in our center. The obesity team in our council includes a surgeon, endocrinologist, dietitian and psychiatrist. Patients are informed about weight loss therapies, several bariatric procedures, and the risks of the interventions. If there is any disagreement among the council members on the decision of surgery of a patient,the surgery is cancelled or postponed.

\section{Before the Scalpel}

A single dose of intravenous cephalosporin is administered for the prophylaxis of surgical site infections. Although guidelines recommend routine antibiotic prophylaxis during bariatric procedures, the evidence belongs to open surgery times. ${ }^{[3-6]}$ To the best of our knowledge, there is no prospective randomized study demonstrating the beneficial influence of antibiotic prophylaxis during laparoscopic bariatric procedures. Further studies are necessary about the necessity, drug selection and dosages of the antibiotic prophylaxis. Contrary to the uncertainty of antibiotic prophylaxis, patients should receive prophylaxis against venous thrombosis with mechanical leg compression devices and low molecular-weight heparin before surgical incision. For mechanical leg compression, thromboembolic socks and sequential compression devices are routinely preferred when available. Preoperative low molecular-weight heparin is carried out routinely before surgery. Anesthesia team places a naso/orogastric tube and arterial line for monitorization. Rapid sequence induction of anesthesia is preferred to decrease gastrointestinal gas which limits the laparoscopic intraabdominal exposure. The proper position of the patient on the operating table during laparoscopic bariatric surgery is vital.
It has been demonstrated that reverse Trendelenburg with the legs flexed at the hips for upper abdominal surgery effectively improves the workspace in obese patients during laparoscopic surgery. ${ }^{[7]}$ While reverse Trendelenburg position moves the intestines and omentum caudally, hip flexion provides some relaxation on the anterior abdominal wall which permits more workspace in the upper abdomen (Figure 1). Fixation of the patient to the operating table with security bandages is mandatory to prevent accidents during positioning. At the beginning of our laparoscopic program, legs open position (surgeon locates between the legs) was being utilized, but later, the patient position was changed to supine and the surgeon was located at the right side of the patient. We found the latter position more practical in our operating room practice.

\section{Before the Stomach}

The pneumoperitoneum is created from the left upper quadrant by a Veress needle and the intraabdominal pressure is arranged to $12-15 \mathrm{mmHg}$. Direct trocar insertion for morbidly obese patients is still a research topic. ${ }^{[8]}$ Direction of the trocar during insertion is more important for morbidly obese patients. If the trocar is not inserted to the abdominal wall in a $90^{\circ}$ angle, the enlarged sub-

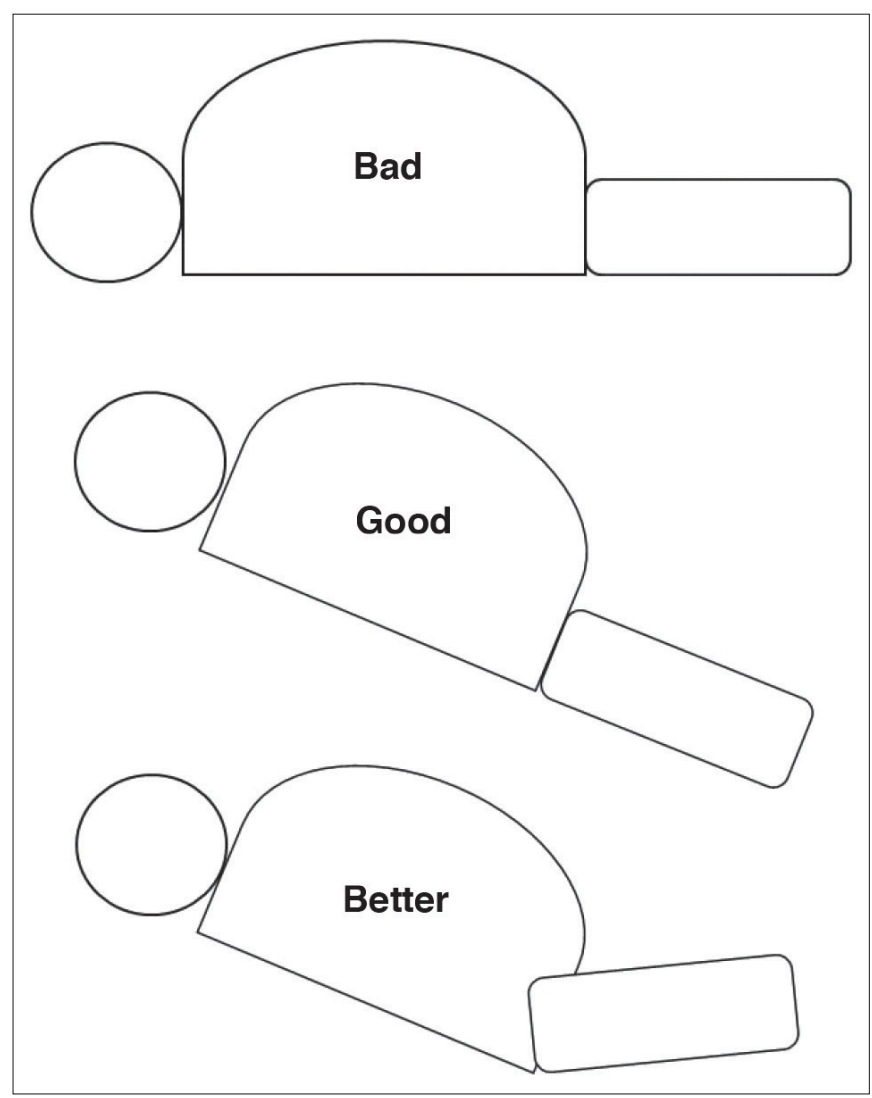

Figure 1. Patient position. 
cutaneous tissue does not let the use of the trocar with multi-directions and can lead to a leak of $\mathrm{CO}_{2}$ to the subcutaneous tissue resulting in massive subcutaneous emphysema (Figure 2). ${ }^{[9]}$ A $12 \mathrm{~mm}$ camera $\left(30^{\circ}\right.$ ) port is placed $21 \mathrm{~cm}$ down and $4 \mathrm{~cm}$ right from the xiphoid process (Figure 3). The umbilicus is not used for trocar placement or as a land mark since it usually results in misplacementin the morbidly obese patients. Two more $12 \mathrm{~mm}$ trocars are inserted from the left and right upper quadrants and all three trocars form a figure of a smiling mouth (Figure 3). Those three trocars should not be placed less than 10 $\mathrm{cm}$ in distance from each other. Two $5 \mathrm{~mm}$ trocars are placed, one just below the xiphoid for liver retraction and the other between the xiphoid, and the $12 \mathrm{~mm}$ right upper quadrant trocars. In order to hang the left liver lobe, Nathanson retractors are used and we wish to see the whitish diaphragm when we fix the retractor (Figure 4, 5). The abdomen is explored for unexpected findings or adhesions. Previous surgeries can fix the omentum and can make it difficult to sweep up. Some surgeons start the

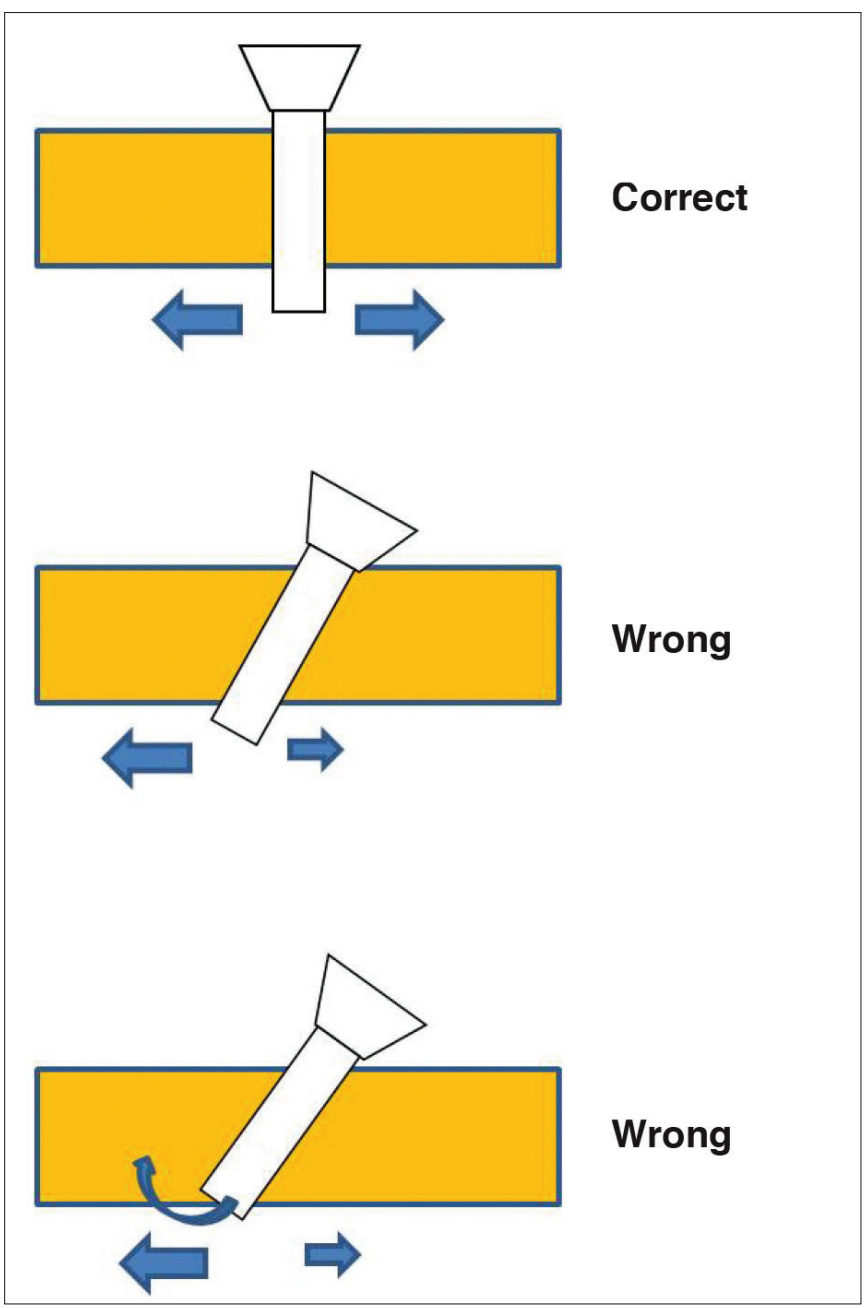

Figure 2. Trocar directions. laparoscopic Roux-en-Y gastric bypass from the intestinal anastomosis and later create the gastric pouch. We prefer the gastric pouch at first and later the intestinal part. This order possibly comes from our open surgical gastric bypass experience.

\section{Gastric Pouch}

The first assistant pulls down the gastric fundus from its most upper part. The left esophago-cardiac ligament is cut and the left diaphragmatic crus is explored (Figure 6). Before leaving the diaphragm, a small marking is put on the inferior site of the liver by cautery to the level of the left crus (Figure 7). The first assistant than pulls the gas-

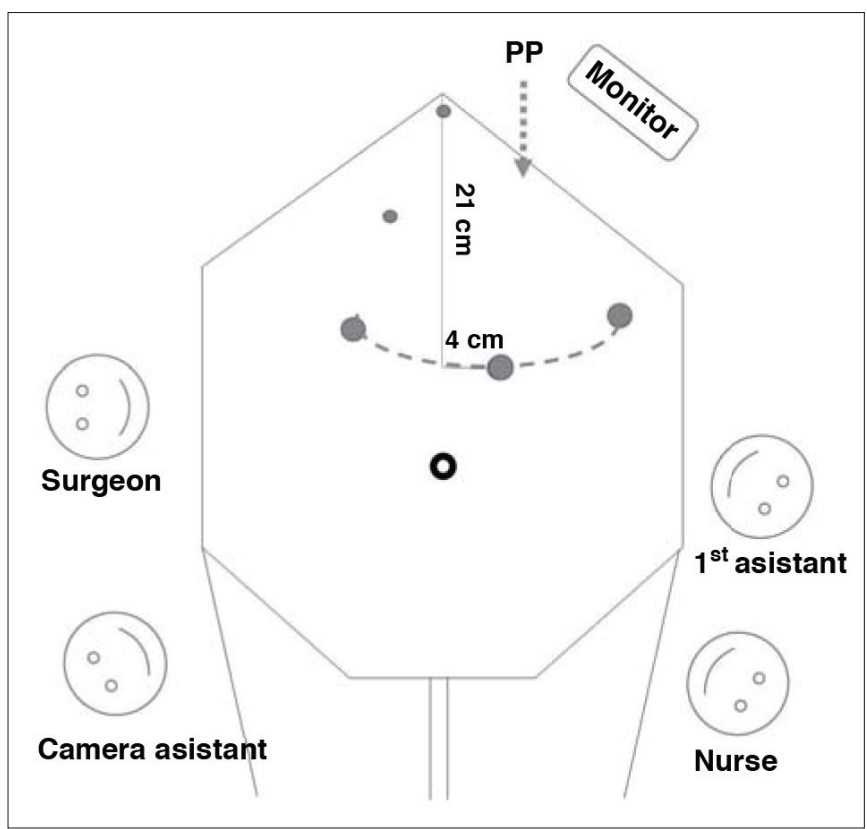

Figure 3. Trocar locations.

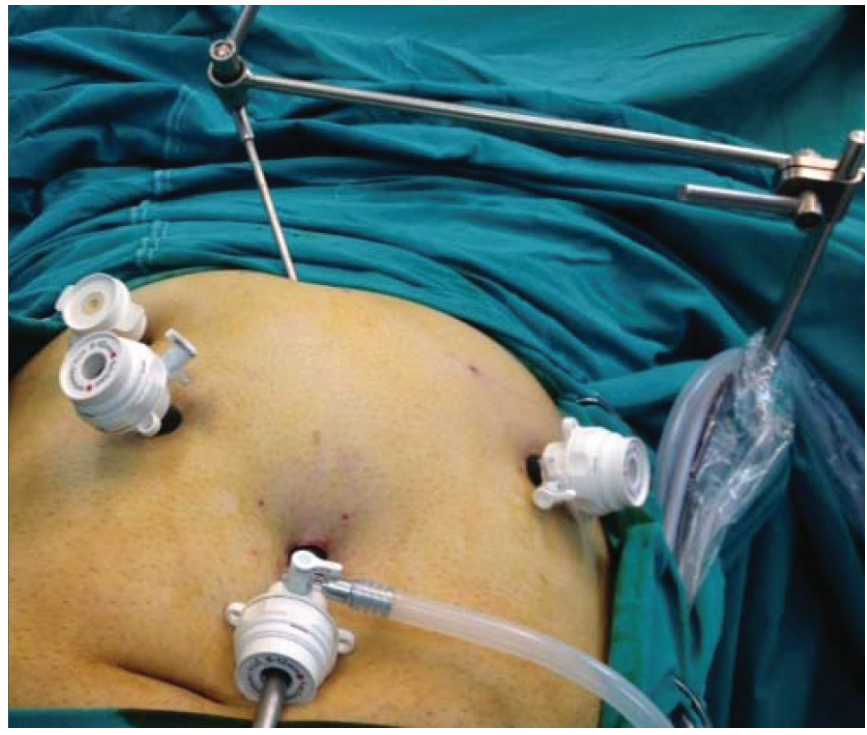

Figure 4. Nathalson liver retractor from outside. 
tric corpus to the lateral with $45^{\circ}$ angle and the surgeon dissects the lesser curvature of the stomach. Energy devices are preferred for the dissection of the lesser. If vis-

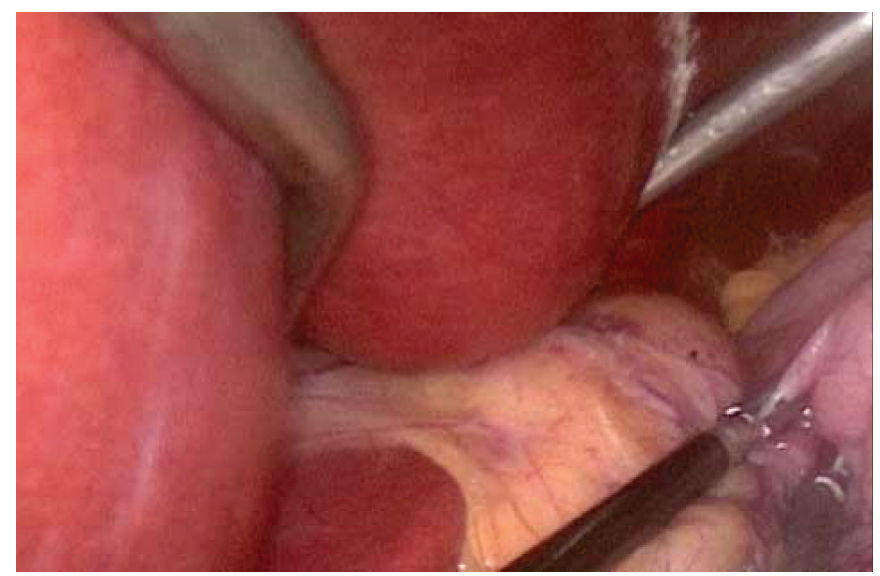

Figure 5. Nathalson liver retractor from inside and whitish diaphragm below the left liver lobe.

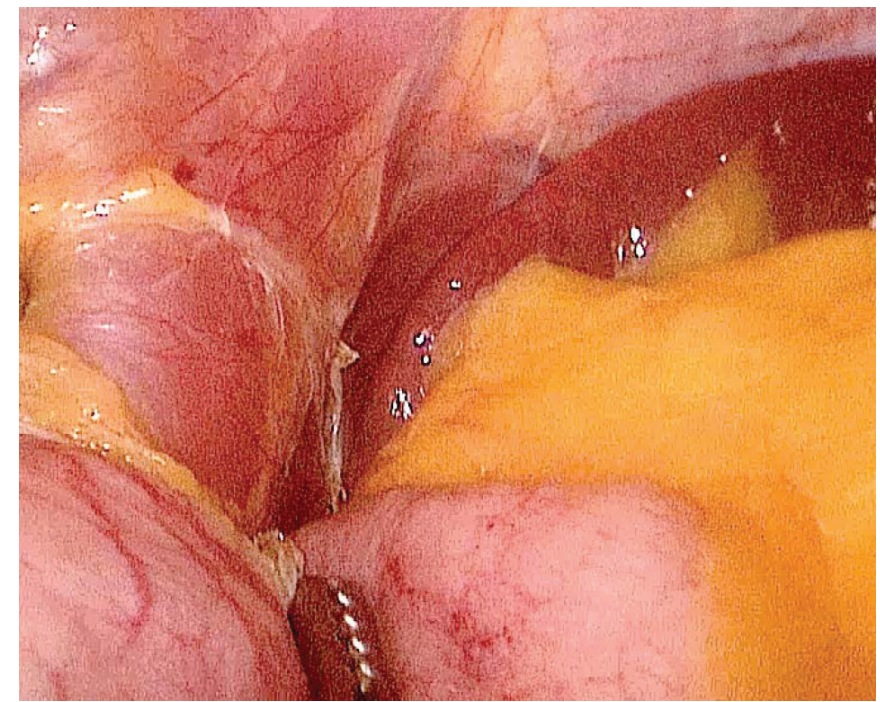

Figure 6. Exploration of left diaphragmatic crus.

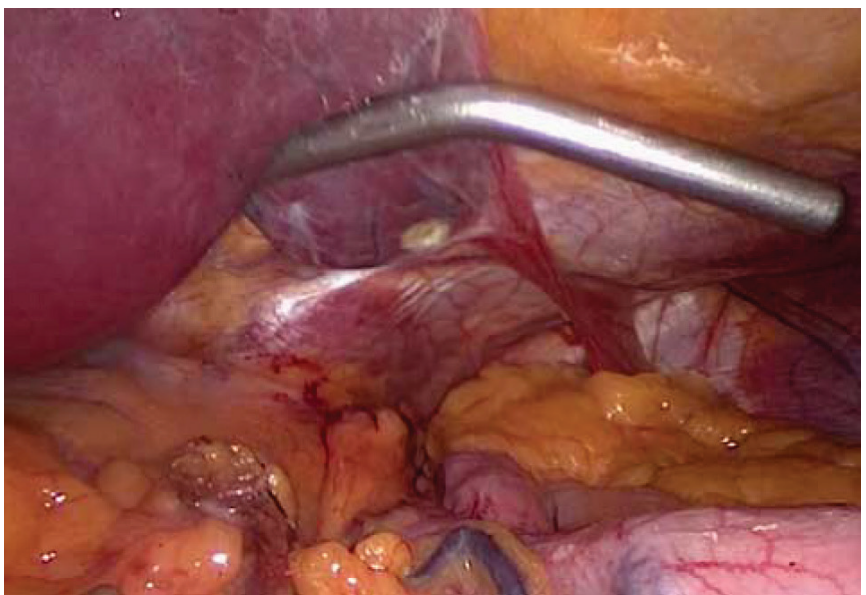

Figure 7. Marking on the liver at the level of left diaphragmatic crus. ible, we prefer the second vein level from the esophagus for transverse transection of the stomach. If the second vein is not visible, we measure $5 \mathrm{~cm}$ from the esophago-

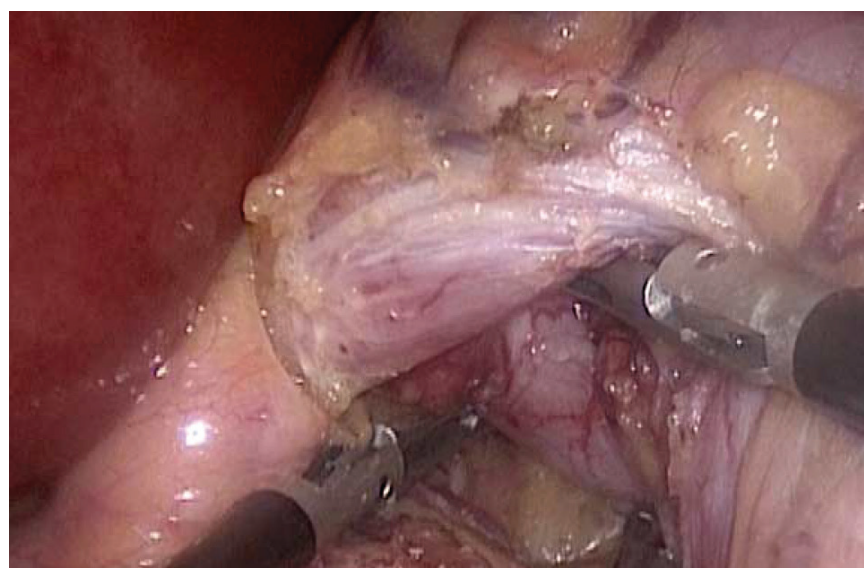

Figure 8. Passing through the posterior of the stomach.

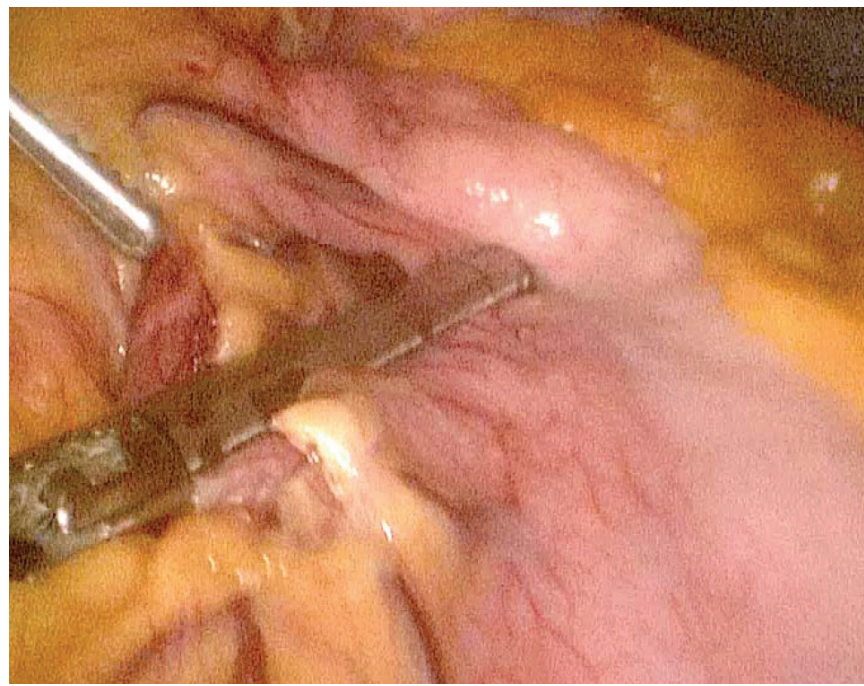

Figure 9. Transverse transaction of the stomach.

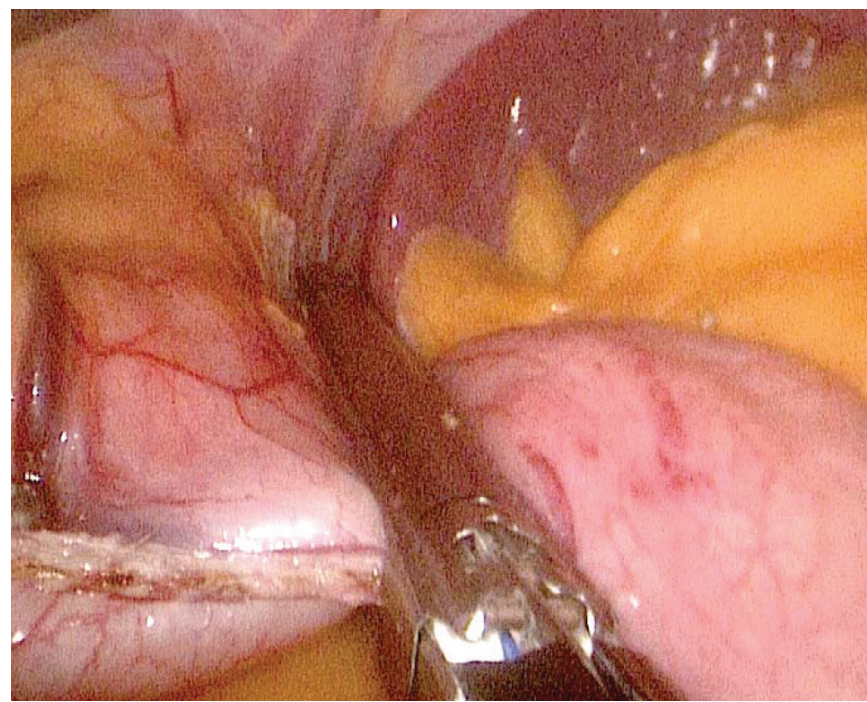

Figure 10. Vertical transaction. 
gastric junction down for gastric transection. We work close to the stomach wall and reach to the posterior space of the stomach (Figure 8). Before the gastric transection, complete with drawal of the oro/nasogastric tube should not be neglected. ${ }^{[10]}$ Nonarticulated $60 \mathrm{~mm}$ blue-cartridge endoscopic staplers have been preferred in all parts of our laparoscopic gastric bypasses (Figure 9). We try to reach the left diaphragmatic crus through the posterior of the stomach. We only cut the fatty tissues at the posterior part of the stomach while moving up, and we named it as "follow the yellow". As firing the second or the third staplers, we pay attention that the tip of the stapler should point out the marking on the liver which reflects the left diaphragmatic crus (Figure 10). For a favorable pouch size, usually one or two vertical stapling should be enough. More stapling means a large pouch or zigzagging of the stapling cartridges (Figure 11).

\section{Finding the Duodenojejunal Junction (Ligamentum Trietz)}

We sweep the omentum up side but we do not divide the omentum routinely. Dividing the omentum was rarely necessary in our practice, and that more studies are required for its necessity. The first assistant retracts up the

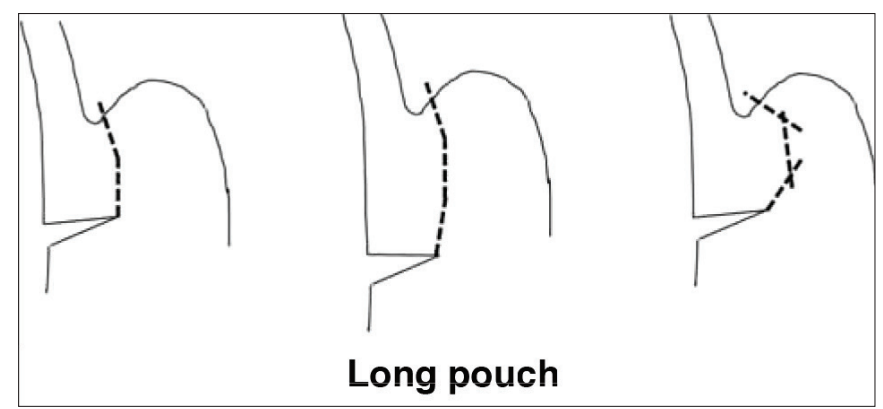

Figure 11. Maximum two vertical stapling.

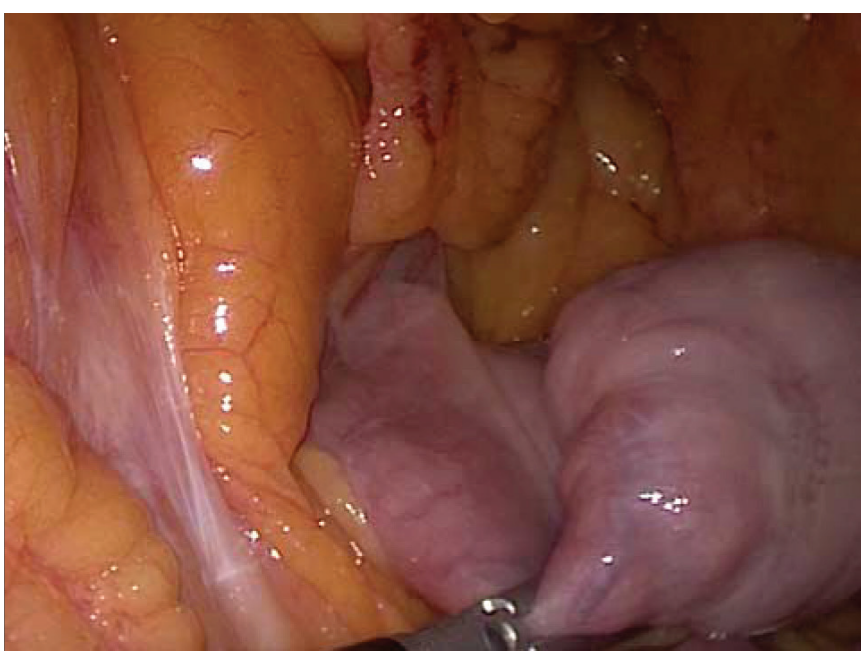

Figure 12. Ligamentum Trietz. transverse colon or its mesocolon, and both hands of the surgeon should be free to search the ligamentum Trietz. The closest small bowel segment to the transverse mesocolon usually indices the ligamentum Trietz (Figure 12). When it is identified, small bowel is followed for $50 \mathrm{~cm}$ distally and these intestinal loops are left to the left side of the abdomen (clockwise follow) (Figure 13).

\section{Gastroenterostomy}

We do all kinds of anastomosis (linear or circular stapler or hand sewn). We suggest anastomosis with linear stapler for the beginners; however, all the others can be the first choice. For linear stapling, we do a gastrostomy (Figure 14) and an enterotomy (Figure 15). The linear stapler is placed to these orifices and fire for the anastomosis (Figure 16). The common orifice of the stapler entrances is closed continuously in two rows with a 3/0 polypropylene suture (Figure 17). For circular stapling, conventional 25

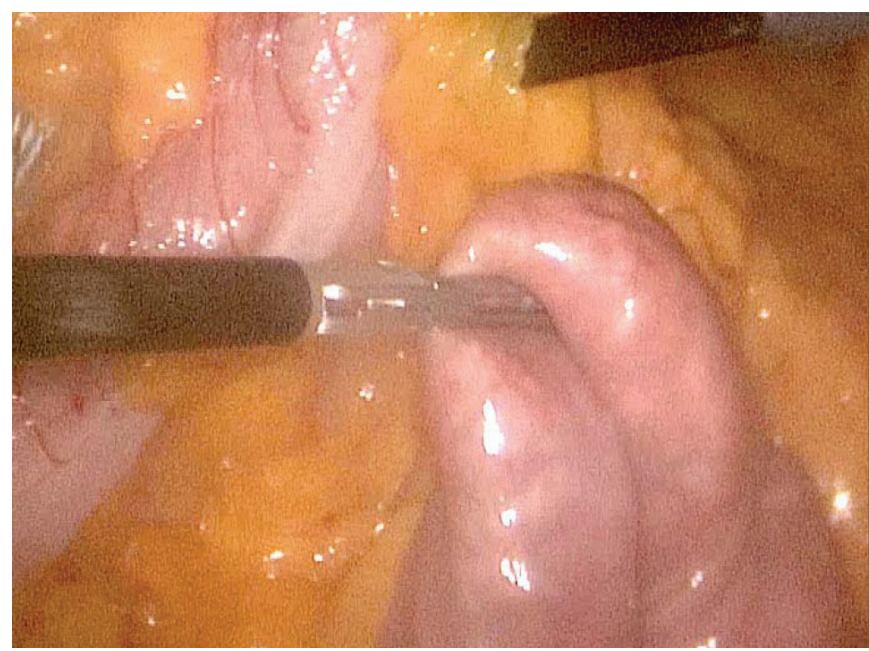

Figure 13. Proximal jejunum.

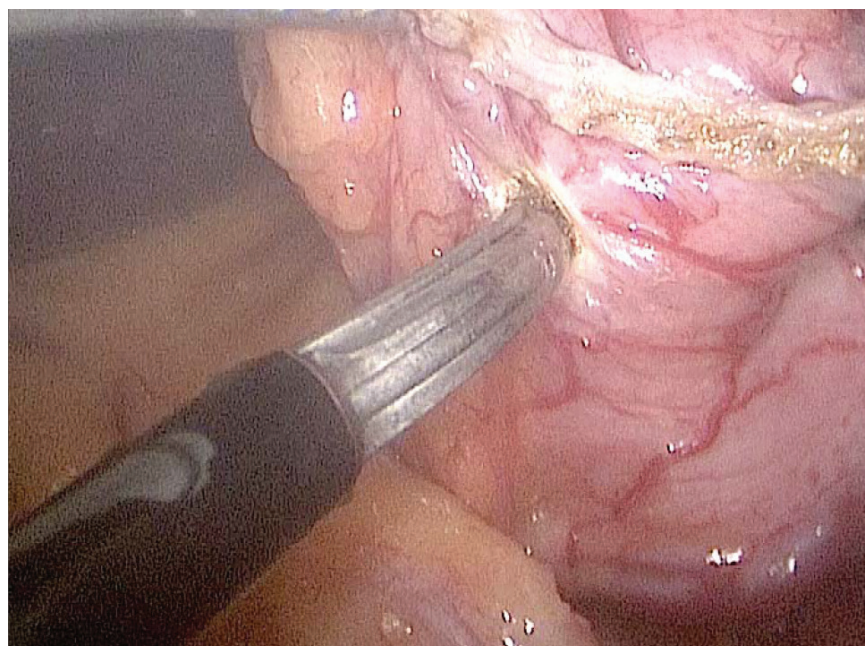

Figure 14. Gastrostomy. 
$\mathrm{mm}$ staplers are preferred not the other ones in which the anvil is passed through the esophagus. Alarger gastrostomyis performed and a purse-string with a 2/0 polyprolene

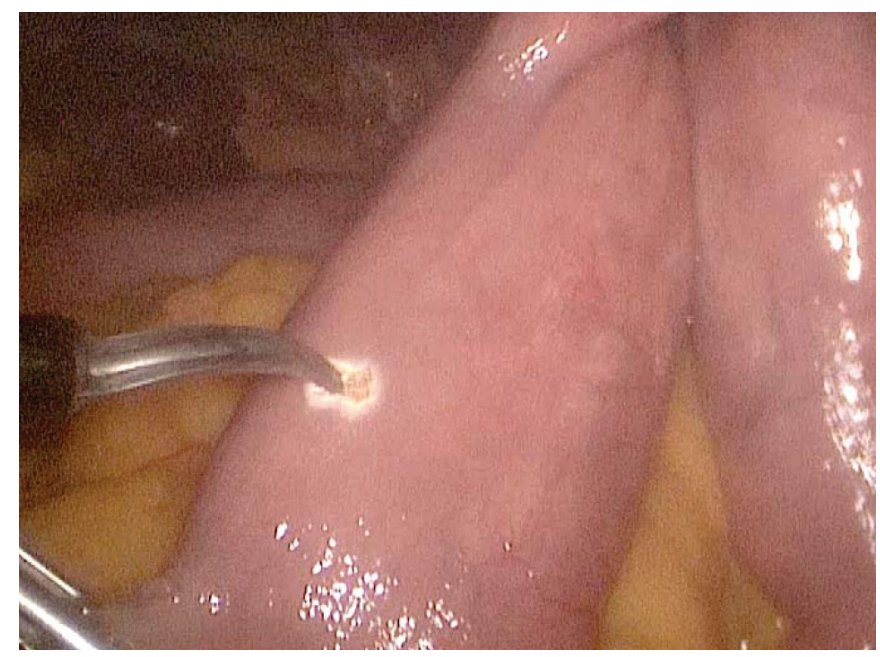

Figure 15. Enterotomy.

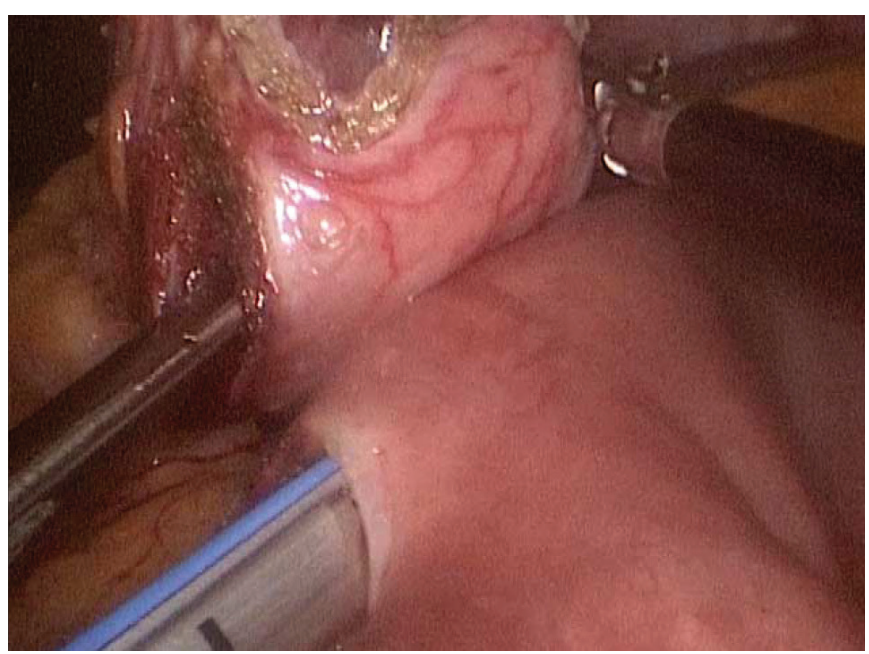

Figure 16. Gastroenterostomy with linear stapler.

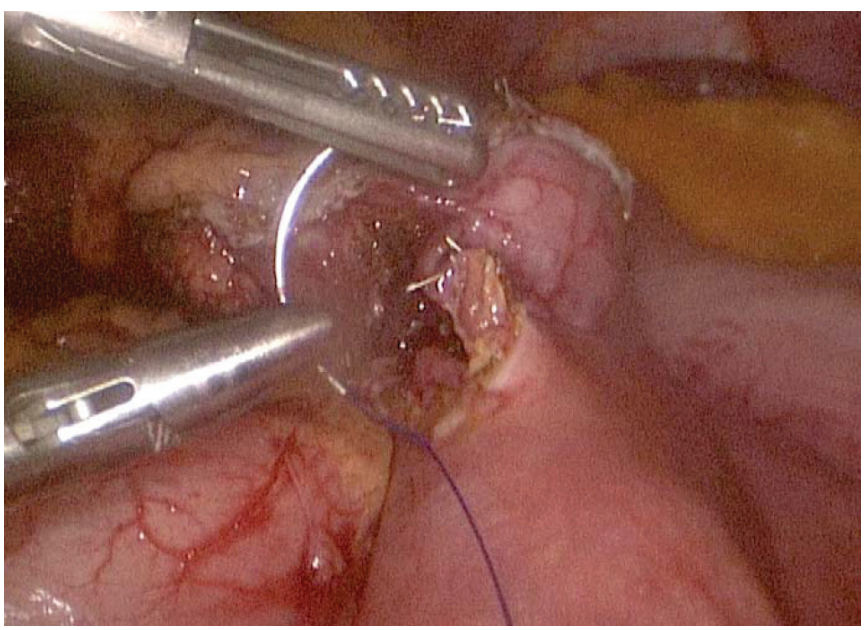

Figure 17. Closing the common orifice of the linear stapler. suture established intra-corporeally (Figure 18). The anvil is inserted through the enlarged left upper quadrant trocar site and placed into the gastrostomy (Figure 19). The

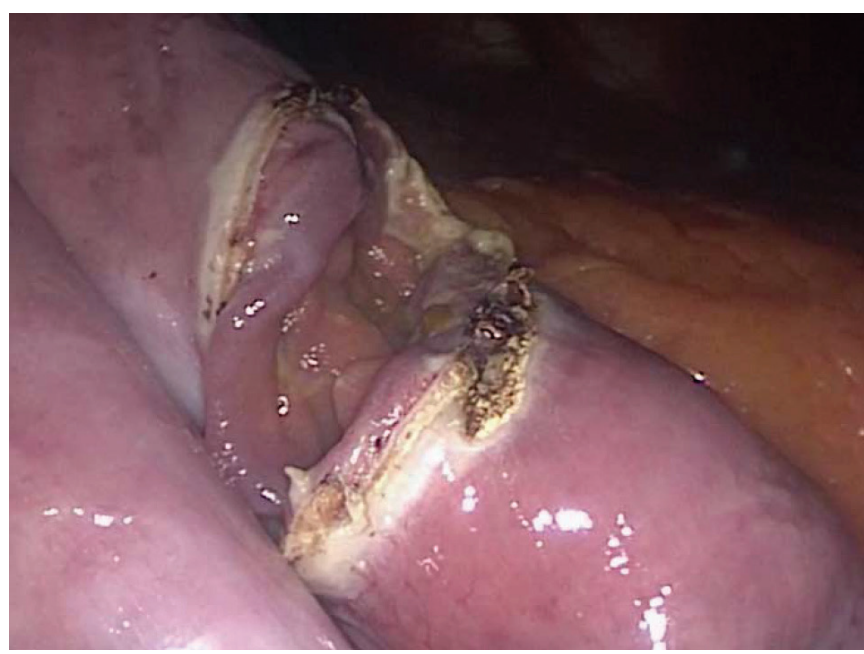

Figure 18. Gastrostomy for circular stapler.

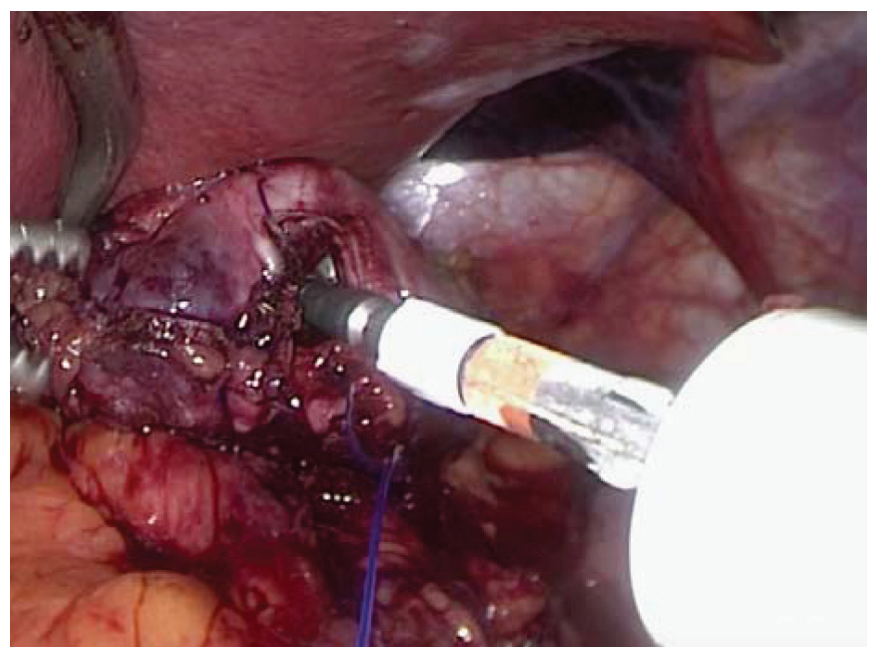

Figure 19. Purse-string suture to the stomach.

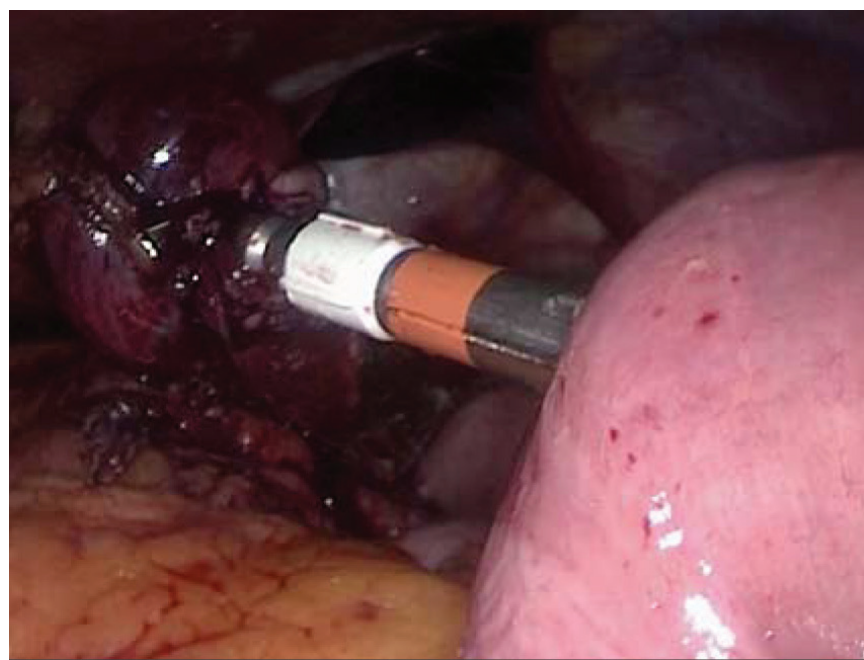

Figure 20. Anastomosis with circular stapler. 
shaft of the circular stapler is inserted through the same opening, connected to the anvil and fired for anastomosis (Figure 20). We compared the results of anastomosis by 21

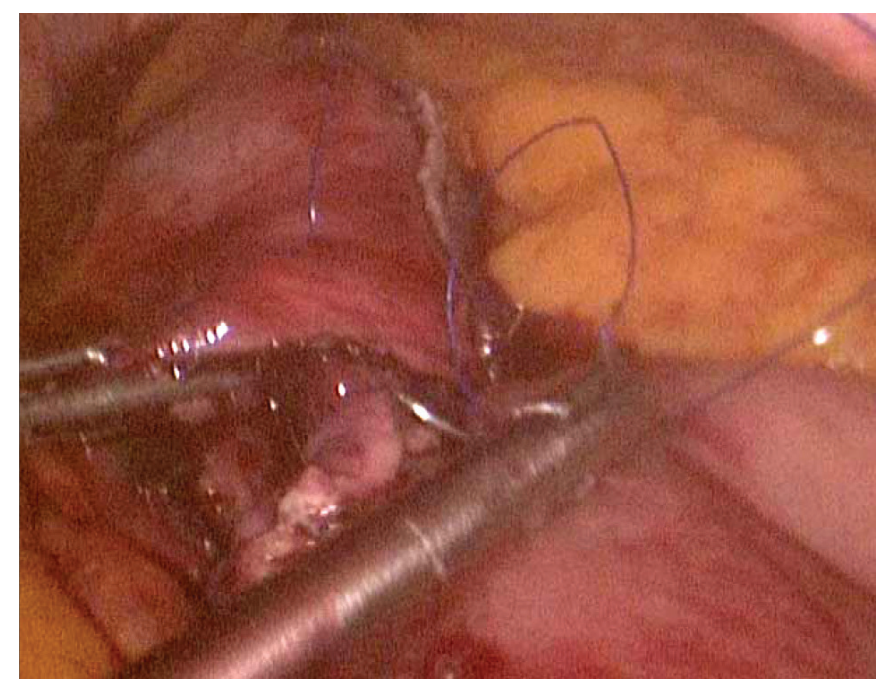

Figure 21. Anastomosis with hand sewn technique.

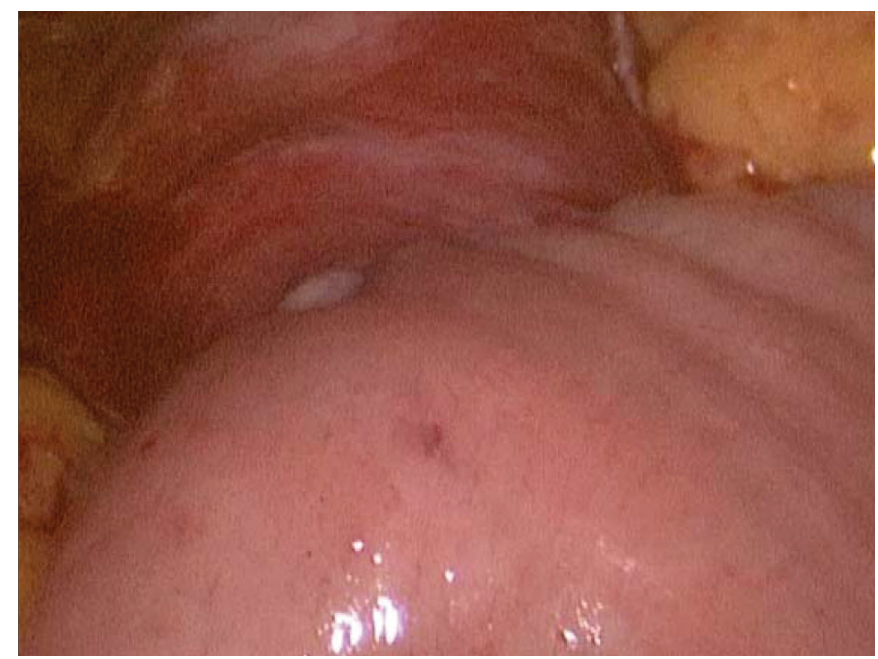

Figure 22. Completed hand sewn gastroenterostomy.

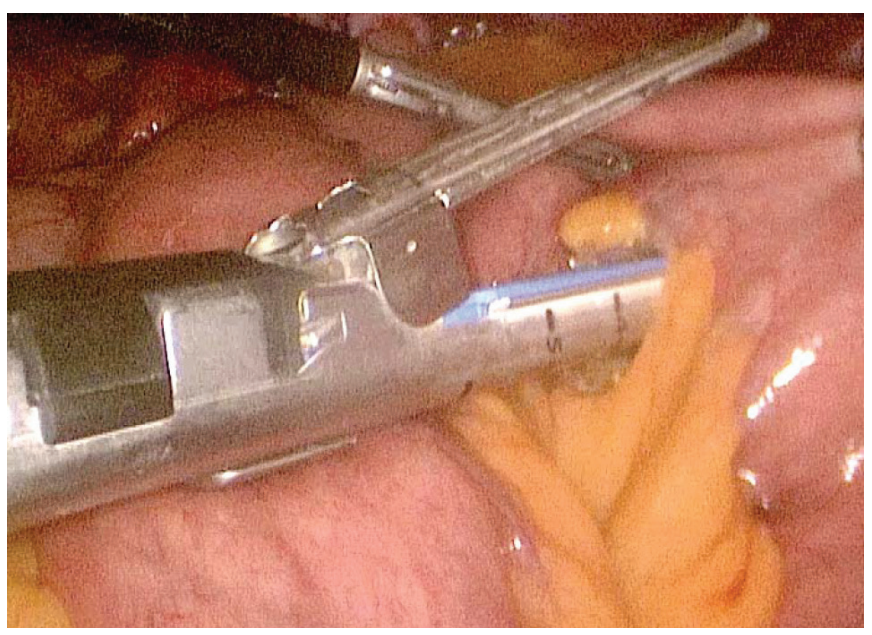

Figure 23. Division of the afferent limb. $\mathrm{mm}, 25 \mathrm{~mm}$ staplers and hand sewn, and found that the $21 \mathrm{~mm}$ stapler group had no advantages with respect to percent of excess weight loss, and they had a higher risk

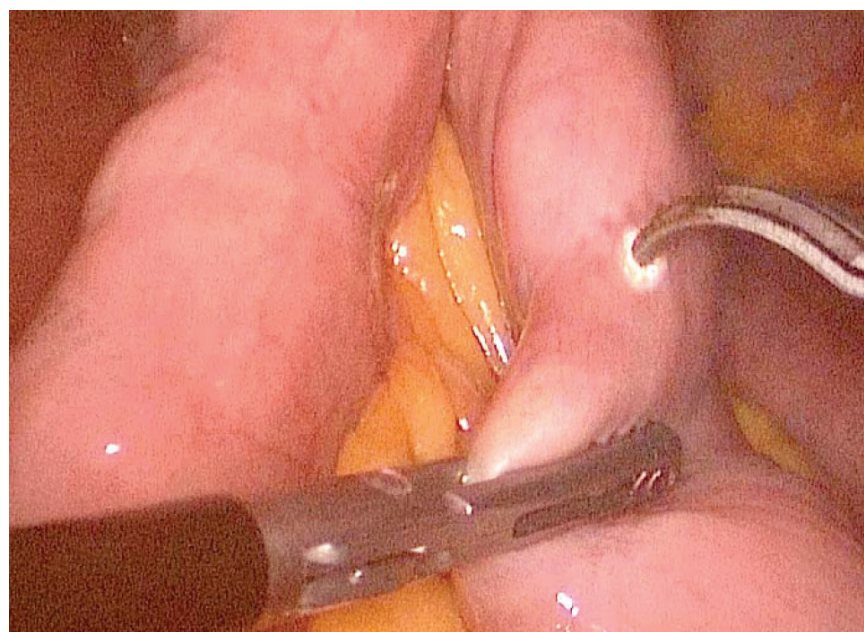

Figure 24. Enterotomy to afferent and efferent intestinal limbs.

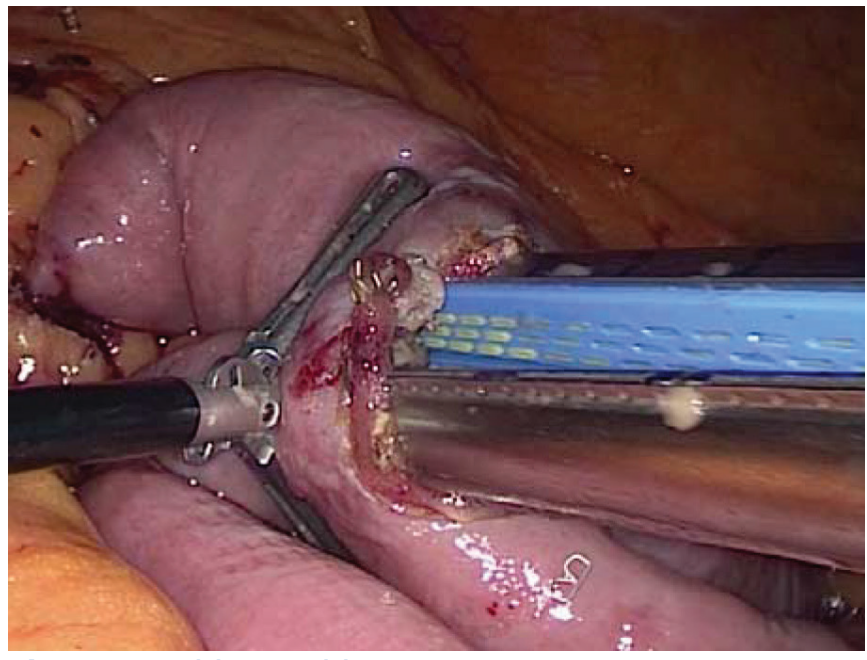

Figure 25. Side-to-side enteroenterostomy.

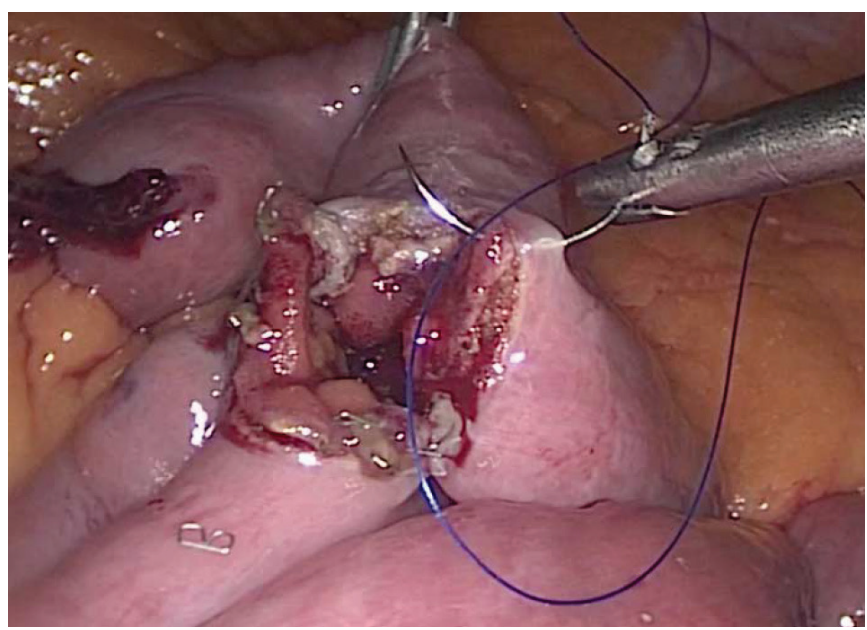

Figure 26. Closing the common orifice. 


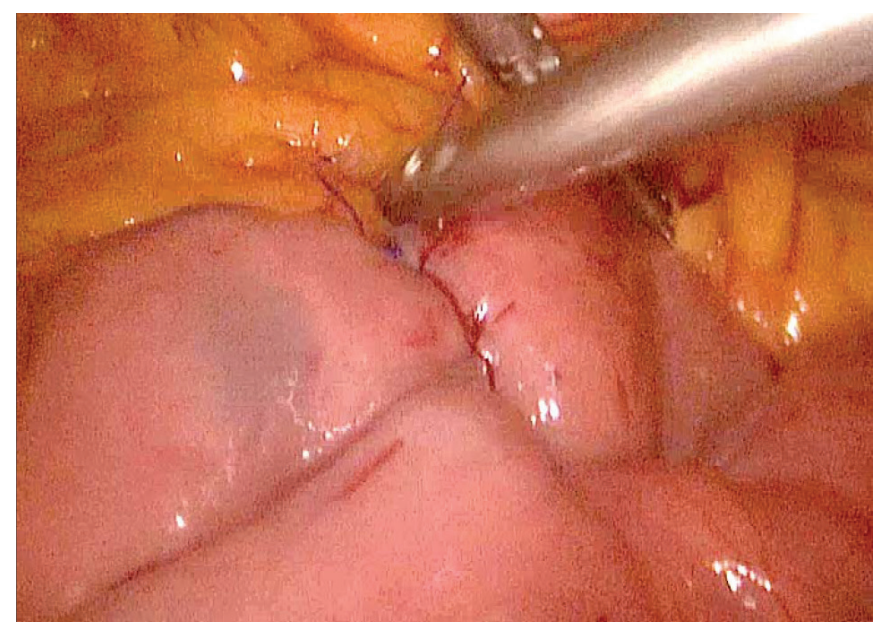

Figure 27. Completed enteroenterostomy.

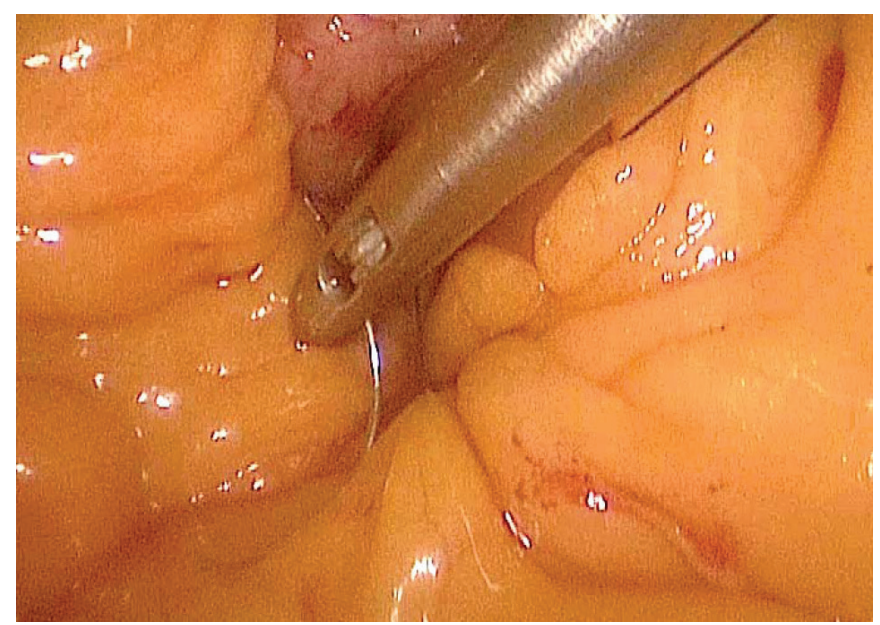

Figure 28. Closing the mesenteric defect.

for dysphagia and endoscopic dilatation. ${ }^{[11]}$ We prefer the hand sewn anastomosis in single row continuously with $3 / 0$ polypropylene suture (Figures 21,22 ).

\section{Distal Anastomosis (Enteroenterostomy)}

Before anastomosis, the afferent limb is divided with a linear stapler (Figure 23). Then, the Trendelenburg position is reversed, the patient turned to left side up and the camera replaced to the right upper quadrant. The efferent limb is followed for $150 \mathrm{~cm}$ and bowel segments are collected at the right side of the patient (counter-clockwise follow). Both efferent and afferent bowel segments are open (Figure 24) and a side-to-side anastomosis is created by a linear stapler (Figure 25). The common opening of the stapler is closed continuously in two rows with $3 / 0$ polypropylene suture (Figures 26, 27).

\section{Almost Finished}

The mesenteric defect of the enteroenterostomy is rou- tinely closed (Figure 28). A methylene blue test is conducted for the gastroenterostomy. Nowadays, abdominal drains are not placed routinely but selectively (39\%). The trocars are removed under laparoscopic guidance and the pneumoperitoneum is evacuated. The orogastric tube is withdrawn.

\section{Postoperative Care}

The patients are moved to intensive care unit and kept there overnight. Patients are mobilized at the sixth hour and start drinking water. They are moved to ward in the morning and discharged on day three if the postoperative course is uneventful. Before discharge, we give them a written instruction for home including hospital control, medications, sutures, diet and considerations for daily life.

\section{References}

1. Linkov F, Bovbjerg DH, Freese KE, Ramanathan R, Eid GM, Gourash W. Bariatric surgery interest around the world: what Google Trends can teach us. Surg Obes Relat Dis 2014;10:533-8.

2. Kayaalp C, Aydin C, Gozeneli O. Preoperative Upper Endoscopy in Patients Undergoing Bariatric Surgery: A Systematic Review. Abstracts from the 18th World Congress of the International Federation for the Surgery of Obesity \& Metabolic Disorders (IFSO), Istanbul, Turkey 28-31 August 2013: p. 405. Obes Surg 2013; 23:1017-243.

3. Fischer MI, Dias C, Stein A, Meinhardt NG, Heineck I. Antibiotic prophylaxis in obese patients submitted to bariatric surgery. A systematic review. Acta Cir Bras 2014;29:209-17.

4. Sauerland S, Angrisani L, Belachew M, Chevallier JM, Favretti F, Finer N, et al; European Association for Endoscopic Surgery. Obesity surgery: evidence-based guidelines of the European Association for Endoscopic Surgery (EAES). Surg Endosc 2005; 19:200-21

5. Pories WJ, van Rij AM, Burlingham BT, Fulghum RS, Meelheim D. Prophylactic cefazolin in gastric bypass surgery. Surgery 1981;90:426-32.

6. Forse RA, Karam B, MacLean LD, Christou NV. Antibiotic prophylaxis for surgery in morbidly obese patients. Surgery 1989;106:750-6.

7. Mulier JP, Dillemans B, Van Cauwenberge S. Impact of the patient's body position on the intraabdominal workspace during laparoscopic surgery. Surg Endosc 2010;24:1398402.

8. Altun H, Banli O, Karakoyun R, Boyuk A, Okuducu M, Onur E, et al. Direct trocar insertion technique for initial access in morbid obesity surgery: technique and results. Surg Laparosc Endosc Percutan Tech 2010;20:228-30.

9. Kayaalp C, Yilmaz S, Aydin C, Piskin T. Massive subcutaneous emphysema involving the head and neck after laparoscopic 
Rouxen-Y gastric bypass. Eur J Surg Sci 2010;1:71-4.

10. Kayaalp C, Aydin C, Gozeneli O. Stapled nasogastric tube during laparoscopic Roux-en-Y gastric bypass: two cases. Abstracts from the 18th World Congress of the International Federation for the Surgery of Obesity \& Metabolic Disorders
(IFSO), Istanbul, Turkey 28-31 August 2013: p. 035. Obes Surg 2013; 23: 1017-1243.

11. Gozeneli O, Kayaalp C. Comparison of gastroenterostomy techniques at Roux-en-Y gastric bypass. Eur J Surg Sci 2013;4:62-7. 